\title{
Solar Panel and Wireless Power Transmission System as a Smart Grid for Electric Vehicles
}

\author{
M. E. Bendib \\ Laboratory of Electrical Engineering and Materials \\ Higher School of Electrical and Energetic Engineering \\ Oran, Algeria \\ bendibmohamed99@yahoo.com
}

\author{
A. Mekias \\ Department of Mathematics \\ Ferhat Abbas University Setif I \\ Setif, Algeria \\ mekiasasma@gmail.com
}

\begin{abstract}
In this work, a smart grid system consisting of solar panels and a battery is presented for an electric or hybrid vehicle. For the integration of green power in our system, solar panels are used to charge the accumulator while the power exchange between the vehicle and the grid is realized by power wireless transfer. This paper presents the design, control, and tests of the wireless power transmission, using the basics of magnetic resonant coupling. Numerical data of the power transfer efficiency of the receiver are presented. Graphs are given to show the comparison of power and efficiency with the distance of the coils. Magnetic and electric models of the system are used to analyze the problem. Using the analysis outcome, a compromise is made to come to an acceptable design achieving both requirements. In particular, appropriate design parameters and resonance frequency are obtained. The analytical results are confirmed by 3D Finite Element Method (FEM) analysis.
\end{abstract}

Keywords-wireless power transmission; smart grid; electric vehicle; magnetic resonance; $3 D$ FEM analysis; solar panel

\section{INTRODUCTION}

A renewable energy Hybrid System (HS) can be applied with grid connection while the owners may be allowed to sell the excessive power to the electric energy provider [1]. The control strategy of an HS is an active research topic [2-4]. Wireless Power Transmission (WPT) is an efficient way for the transmission of electric power through vacuum or atmosphere [5]. By using WPT, power can be transmitted using inductive coupling for short range transmissions, resonant induction for mid-range, and electromagnetic wave power transfer. By using this technology, it is possible to supply power to places that is hard to do with the utilization of conventional wires [5]. Currently, the use of inductive coupling is in development and research phase. The most common wireless power transfer technologies are the electromagnetic induction and the microwave power transfer [6, 7]. For efficient mid-range power transfer, the wireless power transfer system must satisfy three conditions: high efficiency, large air gap, and high power [8]. In general, the microwave power transfer has a low efficiency and can be inefficient for near field power transfer [9]. Wireless power transfer can be done via electric field coupling, which provides an inductively loaded electrical dipole that is an open capacitor or dielectric disk [10]. Extraneous objects may provide a relatively strong influence on electric field coupling.
Magnetic field coupling may be preferred, since extraneous objects in a magnetic field have the same magnetic properties as the empty space.

Electromagnetic induction is applied in short range power transfer. Since magnetic field coupling is a non-radiative power transfer method, it has higher efficiency [11]. However, power transfer range can be increased by applying magnetic coupling with resonance phenomenon applied [12]. A magnetic field is generated when an electric charge moves through space or within an electrical conductor [13]. The geometric shapes of the magnetic flux lines produced by the moving charge (electric current) are similar to the shapes of the flux lines in an electrostatic field [14]. In this work, a smart grid system is used in an electric or hybrid vehicle consisting of solar panels and a battery. The exchange of the power is done by a coaxial WPT system (Figure 1). A mathematical model is used for system analysis, which includes calculation of transferred power, efficiency, coupling coefficient, etc. A design procedure is proposed to achieve high efficiency and transferred power. The work extends the application of low power resonance based on inductive magnetic coupling to high power applications. System parameters suitable for the design specifications are obtained. The analytical results are verified by 3D FEM simulations.

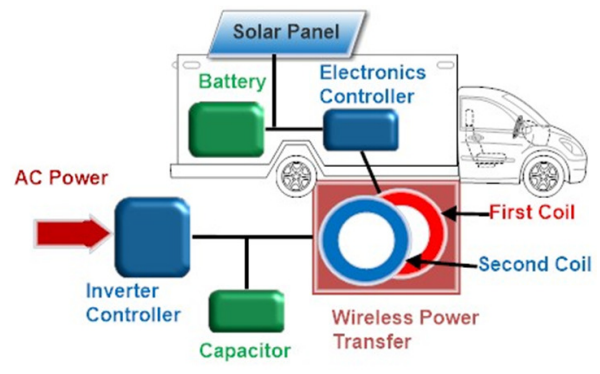

Fig. 1. Electric vehicle compounds as a smart grid

\section{RESEARCH METHOD}

Inductive or magnetic coupling relies on the principle of electromagnetism. When a wire is in the proximity of a magnetic field, an induced magnetic field is created. The inductive coupling is transferring energy between wires 
through magnetic fields. The coaxial wireless system used in our simulation has two parts, the transmitter and the receiver. Each part contains three components, the aluminum frame, the ferrite coil housing, and the Litz wire copper coil. Magnetic resonant coupling uses the same principles with inductive coupling, but it uses resonance to increase the range at which the energy transfer can efficiently take place. Resonance can be of two types: series and parallel. In both types of resonance, the principle of obtaining maximum energy is the same but the methods are quite different.

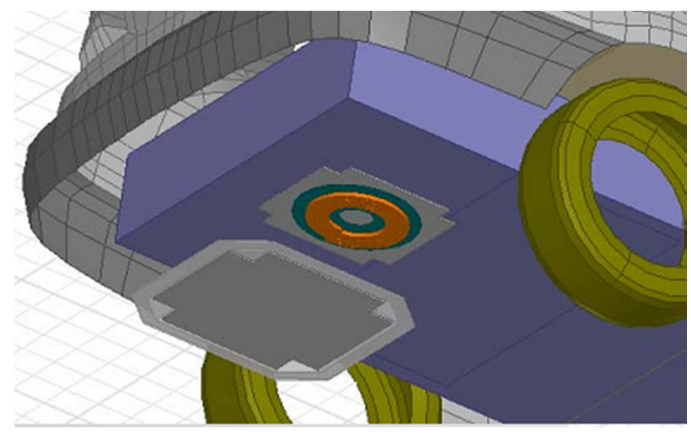

Fig. 2. Coaxial wireless power transfers system

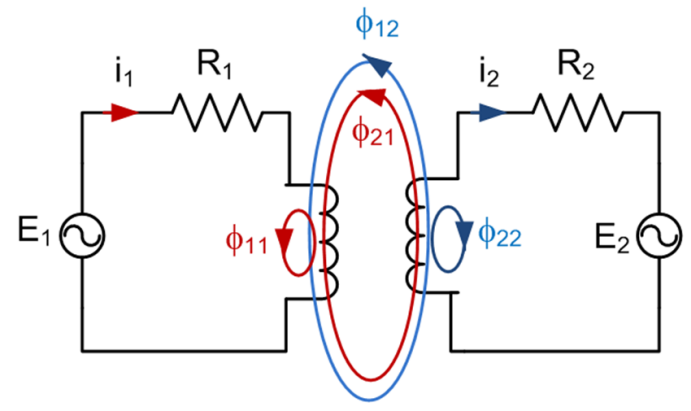

Fig. 3. Equivalent circuit of magnetic coupling

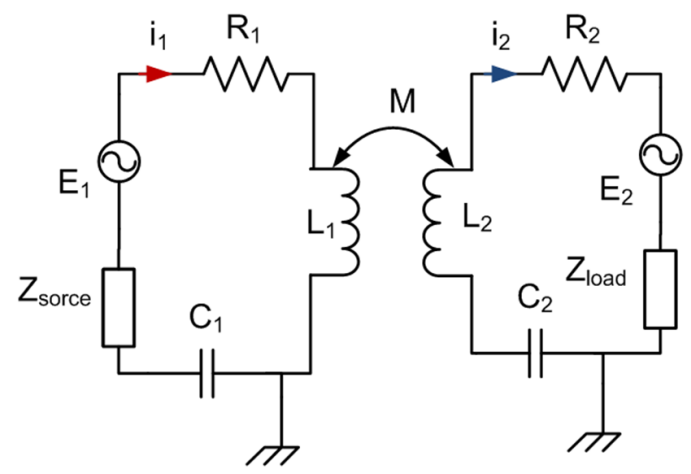

Fig. 4. Equivalent circuit of series resonance.

The quality factor $(Q)$ is a dimensionless parameter that describes the characteristic of a resonator, or equivalently, characterizes a resonator's bandwidth relative to its center frequency. Higher $Q$ indicates a lower rate of energy loss relative to the stored energy of the oscillator, and the oscillations die out more slowly. A system with low quality factor is over damped. Such a system does not oscillate at all, but when displaced from its equilibrium steady state output, it returns to it by an exponential decay, approaching the steady state value asymptotically. Under-damped systems combine oscillations at a specific frequency with decay of the amplitude of the signal. A system with an intermediate quality factor is said to be critically damped. In an over-damped system, the output does not oscillate and does not overshoot its steady-state output. Like an under-damped response, the output of such a system responds quickly to a unit step input.

The efficiency of the coupled system depends on how much energy is transferred from the transmitting to the receiving circuit.

$$
\eta=\frac{W_{\text {rece.max }}}{W_{\text {trans.max }}}
$$

The maximum energy found on the transmitter $W_{\text {trans.max }}$, is the amount of energy initially put on the input capacitor $C$ by the voltage source $E_{1}$

$$
W_{\text {trans.max }}=\frac{1}{2} C E_{1}^{2}
$$

where $C$ acts like a voltage source and completes a series loop with the transmitter elements $R, L_{l}$, and $C_{l}$.

The maximum energy transferred to the receiver is only a fraction of the input energy. The energy found in the receiver circuit is:

$$
W_{\text {recev }}=\frac{1}{2} L_{2} I_{2}^{2}+\frac{1}{2} C_{2} E_{2}^{2}
$$

At maximum voltage level on the receiver circuit, no current flows through the circuit. At this point, the energy stored in the receiver inductor is zero because the current is zero. Thus, the maximized receiver energy is:

$$
W_{\text {trans } \max }=\frac{1}{2} C E_{2 \max }^{2}
$$

\section{DESIGN ANALYSIS}

3D FEM analysis was carried out on a certain resonance frequency to evaluate the design. The 3D FEM model is illustrated in Figure 5.

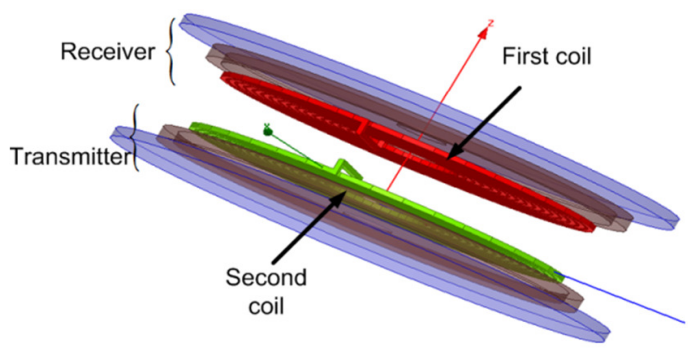

Fig. 5. 3D FEM of the coaxial WPT system

It can be seen in Figure 6(a) that high flux densities appear in and around the air gap, while the flux density in the secondary core remains limited. The magnetic flux vectors are 
depicted in Figure 6(b). It is seen that the vector values are independent from the secondary core length. The leakage flux in the air gap is depicted in Table I showing that it keeps increasing exponentially with the increasing air gap.

(a)

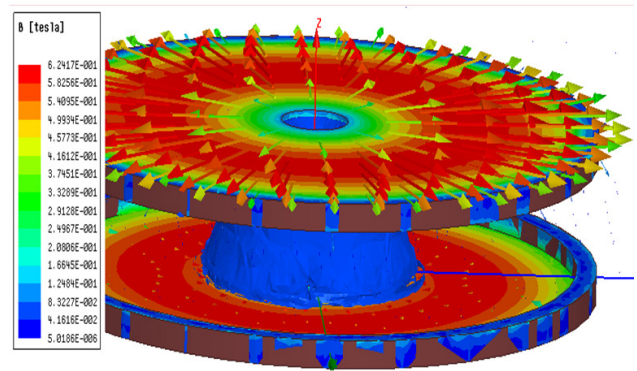

(b)

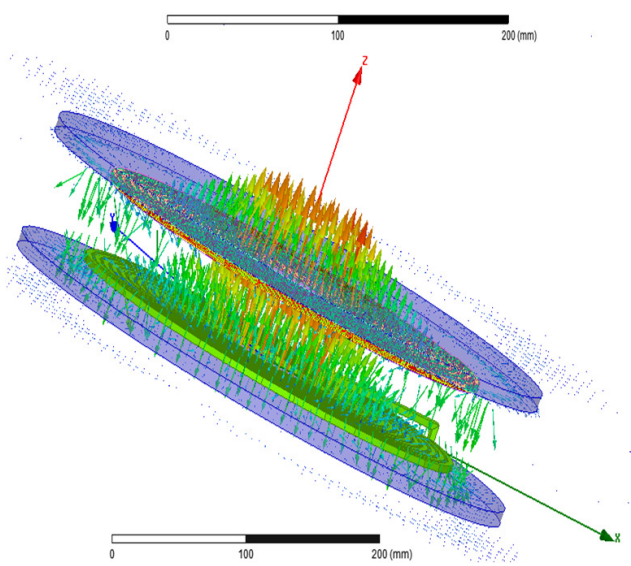

Fig. 6. 3D FEM results: (a) magnetic flux density, (b) magnetic flux vector

TABLE I. WPT MAGNETIC COUPLING COEFFICIENTS

\begin{tabular}{|c|c|c|c|c|}
\hline Distance [mm] & $\boldsymbol{L}_{\boldsymbol{I}}[\mathbf{u H}]$ & $\boldsymbol{M}[\mathbf{u H}]$ & $\boldsymbol{L}_{\boldsymbol{2}}[\mathbf{u H}]$ & Copl. Coef. \\
\hline 5 & 306.7 & 298.9 & 306.7 & 0.9745 \\
\hline 25 & 95.8 & 60.4 & 95.9 & 0.6304 \\
\hline 45 & 82.4 & 28.4 & 82.4 & 0.3450 \\
\hline 65 & 79.9 & 15.0 & 79.9 & 0.1881 \\
\hline 85 & 79.5 & 8.39 & 79.5 & 0.1054 \\
\hline 105 & 79.4 & 4.83 & 79.5 & 0.0608 \\
\hline 125 & 79.5 & 2.85 & 79.55 & 0.0358 \\
\hline 145 & 79.58 & 1.71 & 79.61 & 0.0215 \\
\hline 165 & 79.67 & 1.04 & 79.68 & 0.0131 \\
\hline 185 & 79.82 & 0.64 & 79.79 & 0.0080 \\
\hline 200 & 79.81 & 0.45 & 79.81 & 0.0056 \\
\hline
\end{tabular}

The mutual inductance between the coil transmitter of WPT system and the coil receiver is presented in Figure 7. It can be seen that this factor decreases when the distance increases, which means that the magnetic coupling coefficient is small. For this reason the resonance converter is adopted to obtain maximum power with integrated of solar panels (Figure 8). Figure 8 shows a two side power conversion system. The first (AC-DC-AC) is located in car's park and the second contains a full IGBT bridge single phase with two DC converters (buck and boost). These two sides are linked together by the WPT system, connected to the utility line using a line frequency transformer to offer the rest of the voltage gain.

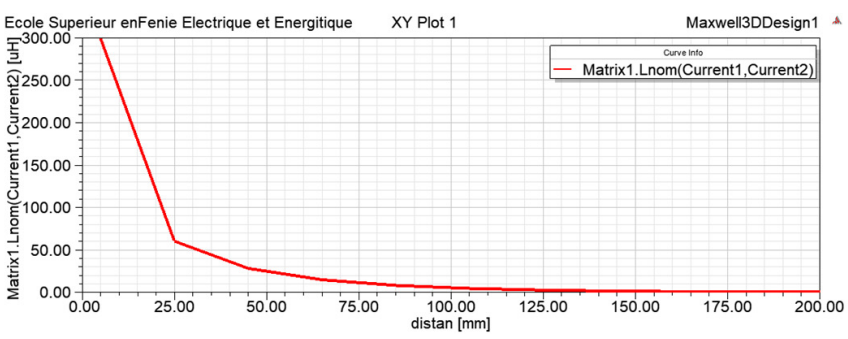

Fig. 7. Mutual inductance for different axial distances.

The choice of the transmitter coil power converter depends on WPT application, power level, and operating frequency. For EVs, the amount of power required to wirelessly charge the battery can range from $3.3 \mathrm{~kW}$ up to several tens of $\mathrm{kW}$, with frequency ranging from $20 \mathrm{kHz}$ up to a few hundreds of $\mathrm{kH}$. For such power and frequency levels, highly efficient $\mathrm{AC}-\mathrm{AC}$ power converters such as full-bridge AC-DC rectifier and DC$\mathrm{AC}$ inverter can be used to drive the transmitter coil. There are several typically used DC-AC power inverters in the $\mathrm{kH}$ frequency range, such as class-E power inverters as they deliver highest efficiencies of all the classes [15]. A singlestage DC-AC power conversion system using a transformerisolated inverter is shown in Figure 9. The system uses line frequency transformers to scale the voltage before connecting to the utility line.

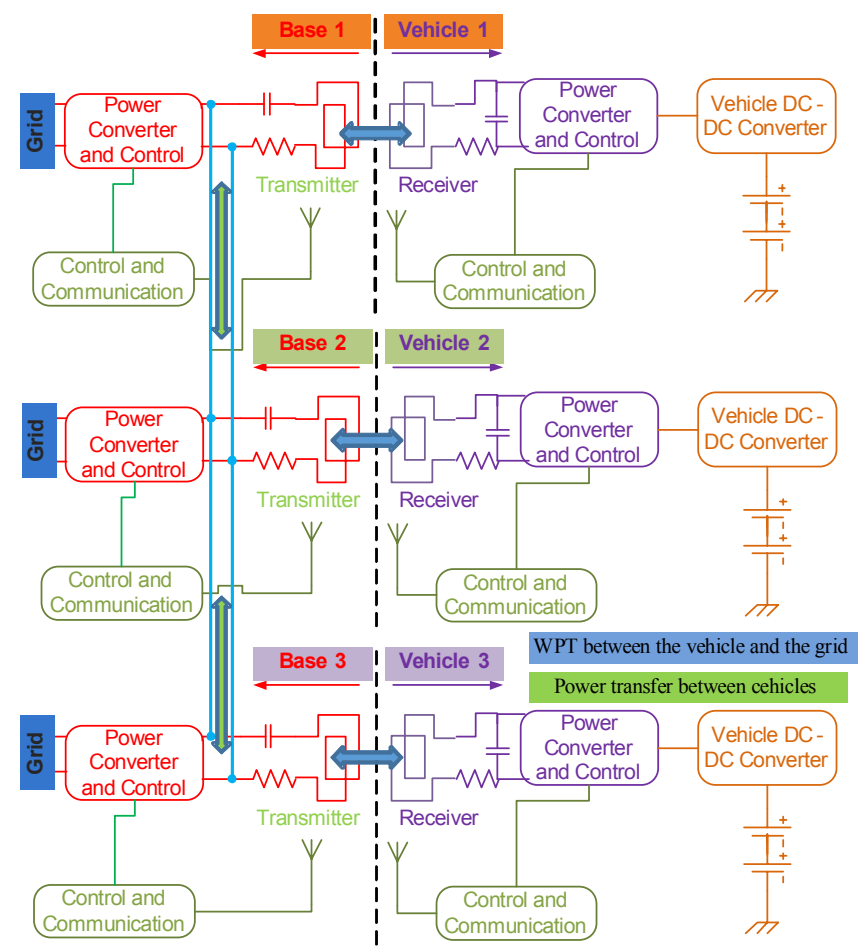

Fig. 8. The block diagram of the WPT system

One two full bridge circuit and series connection of IGBT modules was used. The output ports were connected with an isolation transformer as shown in Figure 8. The PID loop sets the desired DC voltage by using PWM and the PV panels are isolated from the grid at zero-voltage states naturally by the 
WPT system. Power electronics are essential components in a battery power system to convert the varying DC voltage into regulated input DC voltage. A buck converter is employed for charging the vehicle battery for powering all the accessories. The PID controller sets the desired voltage by using PWM. The parameters of the control model are imported to the Simplorer software (Figure 9) from the 3D-FEM WPT model.

(a)
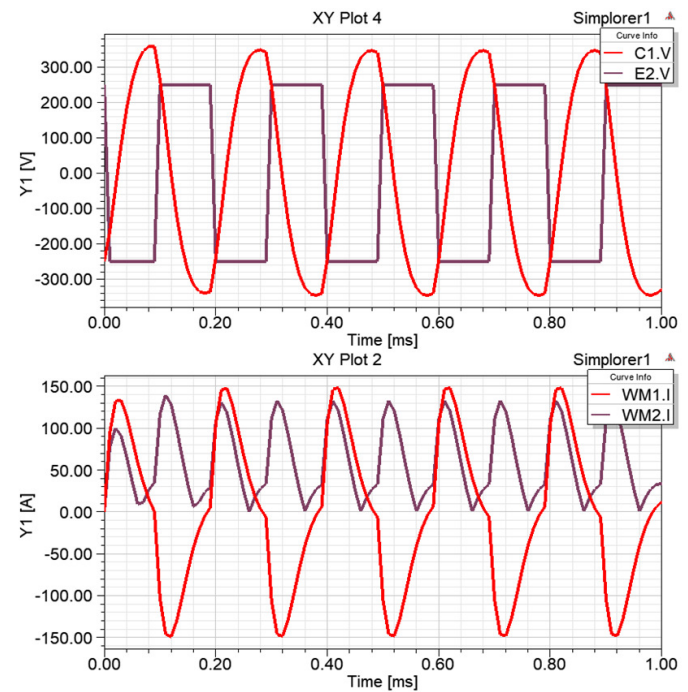

Fig. 9. Simulation results of the WPT system: (a) source and capacitor voltages, (b) current

It is seen that the system efficiency is improved with increasing resonance frequency. The Figures confirm the validity of the analytical results as they are close to the FEM results. It is seen that the FEM results adopt the analytical results in the high power ratings as well. Figure 10 depicts the transmitter system voltage and overall currents system at the frequency of $5 \mathrm{kHz}$. It is seen that the overall coupling coefficient increases with increasing transferred power, and the system efficiency reaches a maximum of $97 \%$. An optimal operating point can be found for every resonance frequency at which transferred power, system efficiency, and overall coupling coefficient are acceptable.

TABLE II. POWER AND EFFICIENCY CALCULATIONS OF THE WTP

\begin{tabular}{|c|c|c|c|}
\hline $\begin{array}{c}\text { Distance } \\
{[\mathbf{m m}]}\end{array}$ & $\begin{array}{c}\text { Transmitted } \\
\text { power }[\mathbf{k W}]\end{array}$ & $\begin{array}{c}\text { Received } \\
\text { power }[\mathbf{k W}]\end{array}$ & Efficiency \\
\hline 5 & 36 & 35 & 97 \\
\hline 25 & 36 & 23 & 63 \\
\hline 45 & 36 & 17 & 34 \\
\hline 65 & 36 & 7 & 18 \\
\hline 85 & 36 & 4 & 10 \\
\hline 105 & 36 & 2.2 & 06 \\
\hline 125 & 36 & 1 & 03 \\
\hline 145 & 36 & 1 & 03 \\
\hline 165 & 36 & 1 & 03 \\
\hline
\end{tabular}

(a)
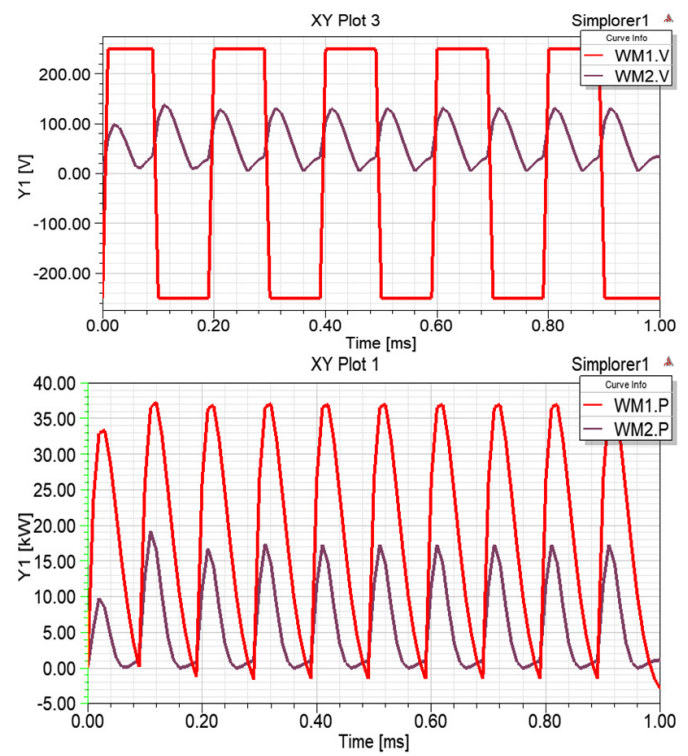

Fig. 10. Simulation results of the WPT system: (a) voltage, (b) power

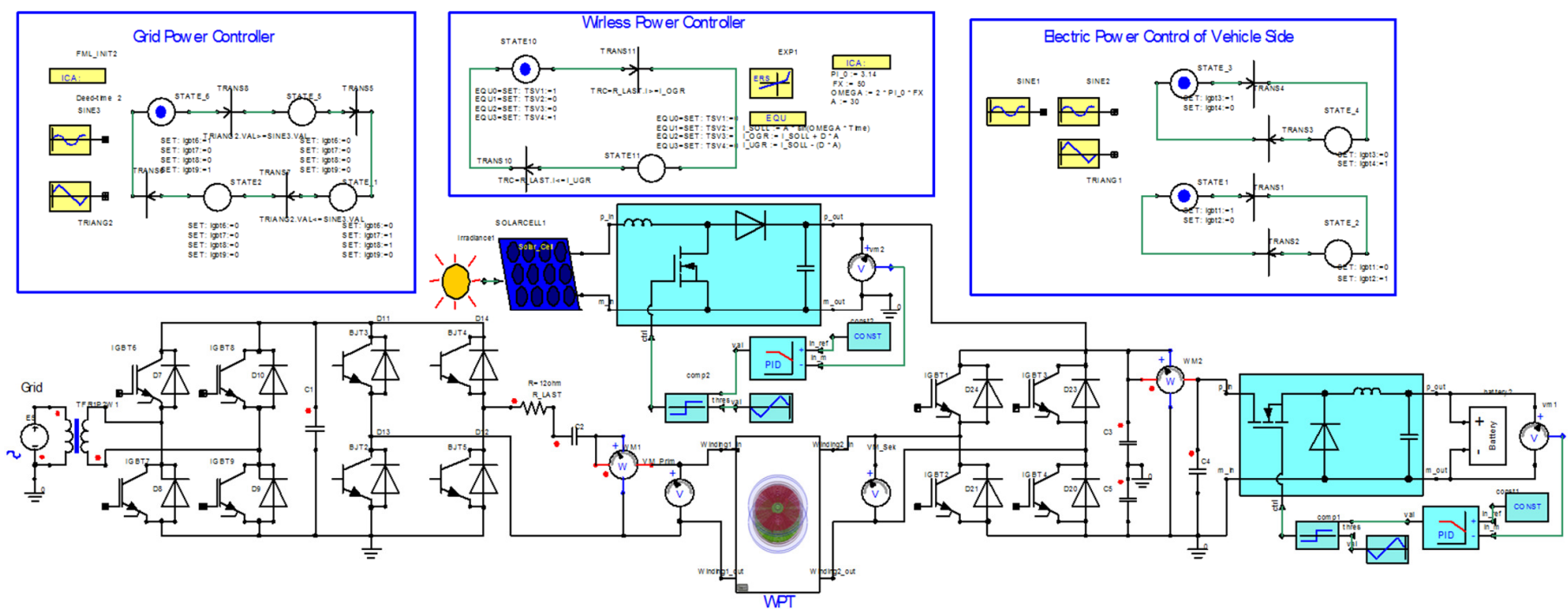

Fig. 11. Multi simulation of PWT system 
(a)

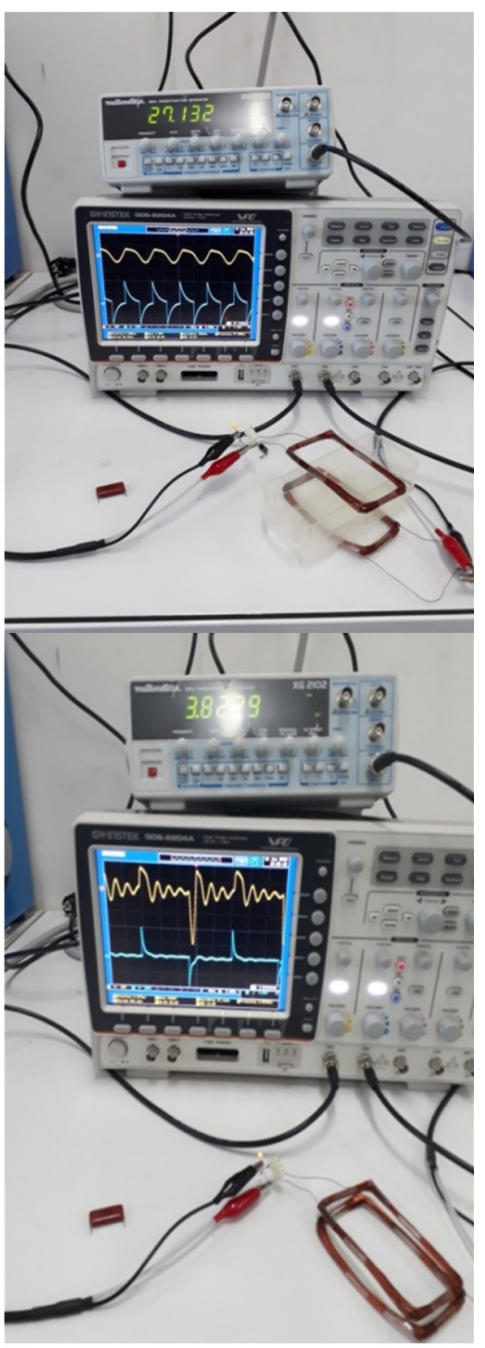

Fig. 12. Reduced project model

After completing the basic device the power efficiency of the two receivers was measured. Transmitting and receiving power were measured and the efficiency was calculated by:

$$
\eta=\frac{P_{r}}{P_{t}} * 100
$$

Power was calculated by:

$$
P=V \times I
$$

\section{RESULTS}

Our experimental realization of the scheme consists of two coils tuned at the same frequency. An oscillation circuit is connected with a Transmitter Coil (TC) which is in turn resonant inductively coupled to a load Receiver Coil (RC). The coils are made of an electrically conducting copper wire wound into a fifty turn coil. Then a radio frequency oscillating signal passed through the TC generating an oscillating magnetic field through the inductance of the TC which is tuned at the same frequency by the inductance of the coil and a resonating capacitor $C$. The $\mathrm{RC}$, tuned at the same resonant frequency receives the power through the magnetic field generated by the TC. For the experiment, the transmitter coil and the receiver coil were constructed using $0.6 \mathrm{~mm}$ radius copper cable. The transmitter circuit mainly consists of the oscillator circuit. A power supply with a rectifier circuit is connected to the transmitter circuit with a coil to transmit the power. The receiver circuit includes only the load coil with an attached capacitor and LED to receive the power.

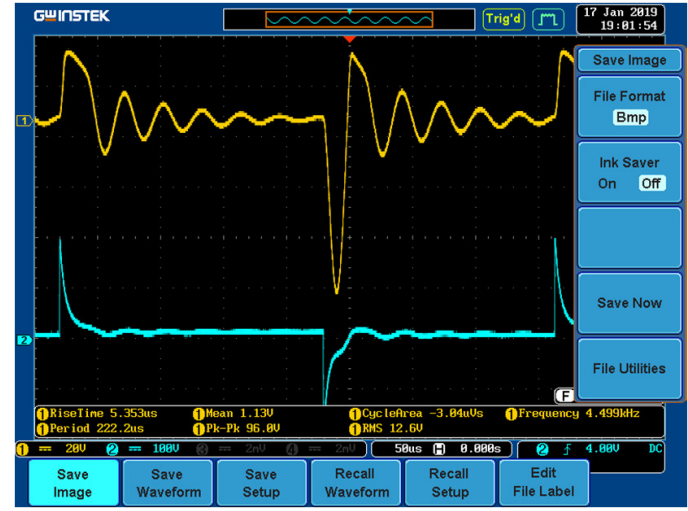

Fig. 13. Reduced project results.

\section{CONCLUSION}

Integrating green power in the power system is very important, so an effort to utilize solar panels to charge the accumulator in hybrid vehicles was conducted. In the same time the power exchange between the vehicles and the grid was realized by PWT. The global control of this system is the main challenge. While presenting magnetic and electric models for a capacitor compensated WPT system, transfer power, efficiency, and coupling coefficient were analyzed. It was observed that the transferred power and efficiency versus resonance frequency follow different trends. At the first glance these two requirements couldn't be achieved at the same frequency. High transferred power is obtained at a low resonance frequency and high efficiency is achieved at a high resonance frequency. Fortunately, a compromise can be reached to achieve acceptable transferred power and high efficiency. The proposed analysis and design can be used as a tool for more accurate system design and optimization.

\section{REFERENCES}

[1] J. B. V. Subrahmanyam, P. Alluvada, Bandana, K. Bhanupriya, C. Shashidhar, "Renewable energy systems: Development and perspectives of a hybrid solar-wind wystem", Engineering, Technology \& Applied Science Research, Vol. 2, No. 1, pp. 177-181, 2012

[2] T. A. Trivedi, R. Jadeja, P. Bhatt, "A review on direct power control for applications to grid connected PWM converters", Engineering, Technology \& Applied Science Research, Vol. 5, No. 4, pp. 841-849, 2015

[3] K. J. Dheeraj, K. Palwalia, "Performance analysis of non-isolated DCDC buck converter using resonant approach", Engineering, Technology \& Applied Science Research, Vol. 8, No. 5,pp. 3350-3354, 2018

[4] V. V. S. K. Bhajana, P. Drabek, "Development and evaluation of an isolated resonant converter for auxiliary power supply in DC traction", Engineering, Technology \& Applied Science Research, Vol. 9, No. 2, pp. 4048-4052, 2019 
[5] A. Kurs, A. Karalis, R. Moffatt, J. D. Joannopoulos, P. Fisher, M. Soljacic, "Wireless power transfer via strongly coupled magnetic resonances", Science, Vol. 317, No. 5834, pp. 83-86, 2007

[6] K. I. Woo, H. S. Park, Y. H. Cho, K. H. Kim, "Contactless energy transmission system for linear servo motor", IEEE Transactions on Magnetics, Vol. 41, No. 5, pp. 1596-1599, 2015

[7] S. L. Ho, J. Wang, W. N. Fu, M. Sun, "A comparative study between novel witricity and traditional inductive magnetic coupling in wireless charging", IEEE Transactions on Magnetics, Vol. 47, No. 5, pp. 15221525,2017

[8] J. L. G. Janssen, J. J. H. Paulides, E. A. Lomonova, "Study of magnetic gravity compensator topologies using an abstraction in the analytical interaction equations", Progress in Electromagnetics Research, Vol. 128, pp. $75-90,2012$

[9] A. Musolino, R. Rizzo, M. Tucci, V. M. Matrosov, "A new passive Maglev system based on Eddy current stabilization", IEEE Transactions on Magnetics, Vol. 45, No. 3, pp. 984-987, 2009

[10] F. Di Puccio, A. Musolino, R. Rizzo, E. Tripodi, "A self controlled Maglev system”, Progress In Electromagnetics Research M, Vol. 26, pp. $187-203,2012$

[11] P. Sergeant, A. Van den Bossche, "Inductive coupler for contactless power transmission", IET Electric Power Applications, Vol. 2, No. 1, pp. 1-7, 2008

[12] L. Peng, O. Breinbjerg, N. A. Mortensen, "Wireless energy transfer through non-resonant magnetic coupling", Journal of Electromagnetic Waves and Applications, Vol. 24, No. 11-12, pp. 1587-1598, 2010

[13] S. Hasanzadeh, S. Vaez-Zadeh, A. H. Isfahani, "Optimization of a contactless power transfer system for electric vehicles", IEEE Transactions on Vehicular Technology, Vol. 61, No. 8, pp. 3566-3573, 2012

[14] X. Luo, S. Niu, S. L. Ho, W. N. Fu, "A design method of magnetically resonanting wireless power delivery systems for bio implantable devices", IEEE Transactions on Magnetics, Vol. 47, No. 10, pp. 38333836,2011

[15] G.A. Kendir, W. Liu, G. Wang, M. Sivaprakasam, R. Bashirullah, M. S. Humayun, J. D. Weiland "An optimal design methodology for inductive power link with class-E amplifier", IEEE Transactions on Circuits and Systems I: Regular Papers, Vol. 52, No. 5, pp. 857-866, 2005 